\title{
ResearchOnline@JCU
}

This is the author-created version of the following work:

Cheong, Z., Moreira, G. M., Bettega, M. H.F., Blanco, F., Garcia, G., Brunger, M. J., White, R. D., and Sullivan, J. P. (2020) A comparison of experimental and theoretical low energy positron scattering from furan. Journal of Chemical Physics, 153 (24) .

Access to this file is available from:

https://researchonline.jcu.edu.au/66580/

(C) The authors. Published under license by AIP Publishing. Accepted Version: Can be made open access in an Institutional Repository after an embargo period of 12 months.

Please refer to the original source for the final version of this work: 


\section{A comparison of experimental and theoretical low energy positron scattering from furan}

Cite as: J. Chem. Phys. 153, 244303 (2020); https://doi.org/10.1063/5.0027874

Submitted: 01 September 2020 . Accepted: 03 December 2020 . Published Online: 22 December 2020

(iD) Z. Cheong, (D) G. M. Moreira, (D) M. H. F. Bettega, (D) F. Blanco, (D) G. Garcia, (D) M. J. Brunger, (iD) R. D. White, and (D) J.P. Sullivan

ARTICLES YOU MAY BE INTERESTED IN

Accurate ground state potential of $\mathrm{Cu}_{2}$ up to the dissociation limit by perturbation assisted double-resonant four-wave mixing

The Journal of Chemical Physics 153, 244305 (2020); https://doi.org/10.1063/5.0028908

Probing the deformation of [12]cycloparaphenylene molecular nanohoops adsorbed on metal surfaces by tip-enhanced Raman spectroscopy

The Journal of Chemical Physics 153, 244201 (2020); https://doi.org/10.1063/5.0033383

Full-dimensional, ab initio potential energy surface for glycine with characterization of stationary points and zero-point energy calculations by means of diffusion Monte Carlo and semiclassical dynamics

The Journal of Chemical Physics 153, 244301 (2020); https://doi.org/10.1063/5.0037175

Challenge us.

What are your needs for periodic signal detection?

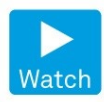

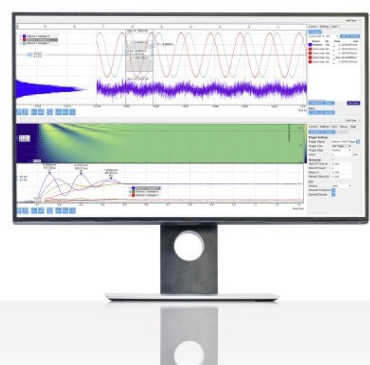

Zurich

Instruments 


\title{
A comparison of experimental and theoretical low energy positron scattering from furan
}

\author{
Cite as: J. Chem. Phys. 153, 244303 (2020); doi: 10.1063/5.0027874 \\ Submitted: 1 September 2020 - Accepted: 3 December 2020 • \\ Published Online: 22 December 2020
}

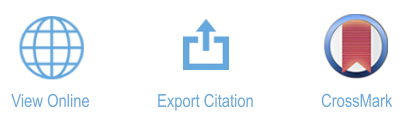

\author{
Z. Cheong, ${ }^{1}$ (D) G. M. Moreira, ${ }^{2}$ (D) M. H. F. Bettega, ${ }^{2}$ (D) F. Blanco, ${ }^{3}$ (D) G. Garcia, ${ }^{4}$ (D) M. J. Brunger, ${ }^{5,6}$ \\ R. D. White, ${ }^{7}$ and J. P. Sullivan ${ }^{1, a)}$ (D)
}

\begin{abstract}
AFFILIATIONS
${ }^{1}$ Laser Physics Centre, Research School of Physics, Australian National University, Canberra, Australia

${ }^{2}$ Departamento de Física, Universidade Federal do Paraná, Caixa Postal 19044, 81531-980 Curitiba, Paraná, Brazil

${ }^{3}$ Departamento de Fisica Atomica, Molecular y Nuclear, Universidad Complutense de Madrid, E-28040 Madrid, Spain

${ }^{4}$ Instituto de Fisica Fundamental, Consejo Superior de Investigaciones Cientificas, Serrano 113-bis, 28006 Madrid, Spain

${ }^{5}$ College of Science and Engineering, Flinders University, Adelaide, SA, Australia

${ }^{6}$ Department of Actuarial Science and Applied Statistics, Faculty of Business and Management, UCSI University, Kuala Lumpur 56000, Malaysia

${ }^{7}$ College of Science and Engineering, James Cook University, Townsville, Qld., Australia
\end{abstract}

a) Author to whom correspondence should be addressed: james.sullivan@anu.edu.au

\begin{abstract}
This paper presents a joint experimental and theoretical study of positron scattering from furan. Experimental data were measured using the low energy positron beamline located at the Australian National University and cover an energy range from $1 \mathrm{eV}$ to $30 \mathrm{eV}$. Cross sections were measured for total scattering, total elastic and inelastic scattering, positronium formation, and differential elastic scattering. Two theoretical approaches are presented: the Schwinger multichannel method and the independent atom method with screening corrected additivity rule. In addition, our data are compared to corresponding electron scattering results from the same target with a number of significant differences observed and discussed.
\end{abstract}

Published under license by AIP Publishing. https://doi.org/10.1063/5.0027874

\section{INTRODUCTION}

In recent years, there has been a push toward developing more sophisticated models of positron interactions with a range of molecules that can be used as templates to build up better descriptions of how positrons interact in biological systems. ${ }^{1-3}$ This work is predominantly driven by the increasingly widespread use of positron emission tomography as a medical imaging technique, particularly for tracking cancer treatment and spread, with a view to better understand the underlying mechanisms responsible for radiation damage. Calculations of even moderately complex molecules require a range of assumptions and approximations to make the problem tractable, so it is imperative that there are high quality experimental data to provide a strict test of accuracy in areas in which theory and experiment overlap. Previous work has included measurements of positron scattering from water, ${ }^{4,5}$ tetrahydrofuran
(THF), ${ }^{6}$ 3-hydroxytetrahydrofuran $(3 \mathrm{~h}-\mathrm{THF}),{ }^{7}$ uracil $^{8}{ }^{8}$ and pyridine.

This paper presents a joint experimental and theoretical study of positron scattering from furan. Furan $\left(\mathrm{C}_{4} \mathrm{H}_{4} \mathrm{O}\right)$ is an organic compound, which is a five-membered aromatic ring consisting of four carbon atoms and one oxygen atom, which can be compared to tetrahydrofuran (THF), a key element of the chain of molecules forming the backbone of DNA. Furan has two double bonds, whereas the carbon ring in THF is entirely singly bonded, and it is interesting to investigate whether the change in structure induces a corresponding change in the scattering properties.

This paper will present experimental measurements of positron scattering from furan from $1 \mathrm{eV}$ to $30 \mathrm{eV}$ and compare the results to two contemporary theories, using the Schwinger multichannel method (SMC) and the Independent Atom Method with Screening Corrected Additivity Rule (IAM-SCAR). 


\section{EXPERIMENTAL METHODS}

Experiments were conducted on the positron beamline at the Australian National University. This apparatus and the data analysis techniques used to determine the cross sections have been previously described in some detail, so only a brief description will be presented here. ${ }^{10-12}$

Positrons are obtained from a radioactive ${ }^{22} \mathrm{Na}$ source, which had an activity of $\sim 740 \mathrm{MBq}$ at the time of the measurements presented here. The source is mounted on a conical holder, which is cooled to $\sim 7 \mathrm{~K}$, allowing the growth of a layer of solid neon. The solid neon moderates the positrons with an efficiency of $1 \%$ (low energy positrons emitted vs positron decay events), resulting in a low energy beam, with an energy width of $1.5 \mathrm{eV}$. This beam is directed into a Surko trap and beam system, ${ }^{10,13}$ which uses a combination of electric and magnetic fields to confine the positrons in an electrode structure, with energy loss coming from collisions with a buffer gas of $\mathrm{N}_{2}$ and $\mathrm{CF}_{4}$. The positrons are trapped and cooled to room temperature in a confining potential well in the last section of the electrode structure. By raising the potential of the confining well, the positrons "spill" over the final electrode in the structure, forming a pulsed beam with an energy resolution of $70 \mathrm{meV}$, full-width at half-maximum (FWHM). The energy of this beam is tunable between $0 \mathrm{eV}$ and $200 \mathrm{eV}$ by adjusting the potential of the final trap electrode.

The pulsed beam is confined in a magnetic field of $0.05 \mathrm{~T}$ and directed to a scattering cell, which contained furan gas for these experiments. The gas sample was obtained from the vapor pressure of a high purity liquid furan sample, which had been cycled through several "freeze-pump-thaw" cycles to eliminate trapped gases. Due to the magnetic field, the positron beam can be considered to have two energy components, $E_{\|}$and $\mathrm{E}_{\perp}$, defined by the motion parallel and perpendicular to (cyclotron motion) the magnetic field, respectively. Initially, the total energy of the beam is predominantly in the $\mathrm{E}_{\|}$component. The pressure of the gas is set to allow for only single collisions with the target, and these collisions induce a change in the energy distribution of the positron beam between $E_{\|}$and $E_{\perp}$ due to angular scattering and energy loss processes. Analysis of the parallel energy distribution after passage through the gas cell can then allow the cross sections to be determined for various scattering processes with normalization through the cell length and pressure within the cell. Positrons scattered backward exit the gas cell and are reflected at the positron trap. They then transit through the gas cell once more with only a small chance of a second collision. As a result, differential cross sections are "folded" around $90^{\circ}$ in the measurement, and a comparison is only made to the calculations after they are similarly folded.

Due to the energy resolution, rotational and vibrational excitations are unable to be resolved in this case, so all elastic scattering is summed over the relevant rovibrational bands. For this paper, "elastic scattering" will refer to this summed cross section. Elastic scattering can, however, be resolved from electronic excitations and ionization by changing the magnetic field at the analyzer after the gas cell, which reduces the energy spread induced by angular scattering, allowing total energy loss to be resolved. ${ }^{11}$ The first electronic state of furan occurs at $6.04 \mathrm{eV},{ }^{14}$ so this technique was employed for the energy range above $6 \mathrm{eV}$ in the current measurements.
TABLE I. Missing forward angle scattering at selected energies. See text for further details.

\begin{tabular}{cc}
\hline \hline Energy $(\mathrm{eV})$ & Angle (deg) \\
\hline 3 & 10.5 \\
5 & 8 \\
7 & 15.5 \\
10 & 13 \\
15 & 10.5 \\
\hline
\end{tabular}

Because of the strong magnetic field confinement, a given energy spread can be mapped to an angular resolution. This affects the elastic scattering measurements, giving a minimum angle below which the unscattered and scattered positrons are indistinguishable. In a molecule such as furan, with a permanent dipole moment of 0.69 $\mathrm{D},{ }^{15}$ this can lead to a significant underestimation of the grand total and total elastic cross sections, ${ }^{16}$ as the target dipole moment leads to a large amount of forward angle scattering and rotational excitation. The minimum angle included in the measurements can be calculated from the measured angular resolution, and the relevant angles are tabulated in Table I. Note that above $6 \mathrm{eV}$, changing the magnetic field at the analyzer also degrades the angular resolution somewhat. In this case, care needs to be taken when comparing the experimental results to a theoretical calculation of grand total or total elastic cross sections, which includes all scattering angles. Correcting the experimental data to account for the missing angles is problematic, as it assumes a known differential cross section (DCS) shape, which can typically only be gleaned from theory. The preferred method of comparison (at least by the present authors) is to compare to an adjusted calculation, which is integrated over only those scattering angles included in the measurement (given in Table I in the present case).

Experimental data are normalized to absolute values through the measurement of the gas pressure and the scattering cell length, as described previously. ${ }^{10,11}$ Errors are determined through a combination of systematic errors in these values as well as the statistical error arising from the measurement with the total error presented for all measurements here. For most cases in the present data, the uncertainty is dominated by the statistical error.

In this paper, measurements are presented for the grand total, the total elastic, and the total positronium (Ps) formation cross sections. We have also measured the total inelastic scattering cross sections, which include ionization and electronic excitation processes. Differential elastic scattering cross sections are additionally presented for energies from $3 \mathrm{eV}$ to $15 \mathrm{eV}$. There are no other positron scattering data available for this target, as demonstrated in a recent review of positron scattering from molecular targets.

\section{THEORETICAL METHODS}

\section{A. Schwinger multichannel method}

We employed the SMC method, which is a variational approach to the scattering amplitude, to compute elastic cross sections for 
scattering of positrons by the furan molecule. Its implementation has previously been described precisely, ${ }^{18,19}$ so here we only describe the most relevant aspects of the method for the present calculations.

In the SMC method, the working expression for the scattering amplitude in the molecular frame is written as

$$
f\left(\vec{k}_{f}, \vec{k}_{i}\right)=-\frac{1}{2 \pi} \sum_{\mu, v}\left\langle S_{\vec{k}_{f}}|V| \chi_{\mu}\right\rangle\left(d^{-1}\right)_{\mu v}\left\langle\chi_{v}|V| S_{\vec{k}_{i}}\right\rangle
$$

where

$$
d_{\mu v}=\left\langle\chi_{\mu}\left|A^{(+)}\right| \chi_{v}\right\rangle
$$

and

$$
A^{(+)}=Q \hat{H} Q+P V P-V G_{P}^{(+)} V .
$$

In these expressions, $\left|S_{\vec{k}_{i}}\right\rangle$ is a solution of the unperturbed Hamiltonian $H_{0}$ (the kinetic energy operator for the incident positron plus the target Hamiltonian) and it is obtained as a product of a target state and a plane wave; $V$ is the interaction potential between the incident positron and the molecular target; $\left|\chi_{\mu}\right\rangle$, with $\mu=(a, m)$, is a configuration state function (CSF), i.e., an $(N+1)$-particle variational trial function, which is the product of a target state (labeled a) and a positron scattering orbital (labeled $m$ ), used in the expansion of the trial scattering wave function (see below); $\hat{H}=E-H$ is the total energy of the collision minus the full Hamiltonian of the system, where $H=H_{0}+V ; P=\sum_{\ell \in \text { open }}\left|\Phi_{\ell}\right\rangle\left\langle\Phi_{\ell}\right|$ is a projection operator onto the open-channel space defined by the target electronic states (with $a=\ell) ; Q=(\mathbb{1}-P)=\sum_{r \in \text { closed }}\left|\Phi_{r}\right\rangle\left\langle\Phi_{r}\right|$ is the projector onto the closed electronic channels of the target (with $a=r)$; and $G_{P}^{(+)}$is the free-particle Green function projected onto the $P$-space.

In the present application, we considered only elastic scattering, so $P=\left|\Phi_{0}\right\rangle\left\langle\Phi_{0}\right|$, where $\left|\Phi_{0}\right\rangle$ represents the ground state of the target molecule obtained at the Hartree-Fock (HF) level. The elastic cross sections were obtained within the static plus polarization (SP) approximation, where the scattering wave function is expanded in a set of CSFs as follows:

$$
\left|\Psi_{\vec{k}_{i}}^{(+)}\right\rangle=\sum_{m} c_{0 m}^{(+)}\left(\vec{k}_{i}\right)\left|\chi_{0 m}\right\rangle+\sum_{r} \sum_{m} c_{r m}^{(+)}\left(\vec{k}_{i}\right)\left|\chi_{r m}\right\rangle,
$$

where $\left\{c_{a m}^{(+)}\left(\vec{k}_{i}\right)\right\}$ is a set of variational coefficients. The static term corresponds to the first term on the right-hand-side (RHS) of the above equation, and the polarization term corresponds to the second term of the RHS of this equation. The CSFs, $\left|\chi_{0 m}\right\rangle$ and $\left|\chi_{r m}\right\rangle$, are constructed as

$$
\left|\chi_{0 m}\right\rangle=\left|\Phi_{0}\right\rangle \otimes\left|\varphi_{m}\right\rangle
$$

and

$$
\left|\chi_{r m}\right\rangle=\left|\Phi_{r}\right\rangle \otimes\left|\varphi_{m}\right\rangle,
$$

where $\left|\varphi_{m}\right\rangle$ is a single-particle orbital used to represent the positron scattering orbital and $\left|\Phi_{r}\right\rangle$ is a singly excited state of the target obtained from a virtual excitation of the target out of the HF reference state. Multichannel scattering, or flux competition, effects have been shown to be important in previous electron scattering calculations, ${ }^{20,21}$ and we would expect that to also be the case in attempts to provide accurate calculations of positron scattering. As a consequence, the restriction of these calculations to just the elastic scattering channel can be expected to lead to limitations in the present SMC cross sections.

The target ground state geometry was optimized at a second order Møller-Plesset (MP2) level with the TZV++(3d, 1p) Cartesian-Gaussian basis set, as implemented in the General Atomic and Molecular Electronic Structure System (GAMESS) package. ${ }^{22}$ The optimized geometry was used in scattering calculations. To take the polarization effects into account, we employed modified virtual orbitals (MVOs) ${ }^{23}$ obtained from a cationic Fock operator with charge +6 to represent the particle and scattering orbitals. For the construction of the configuration space, we considered single excitations from the hole (occupied) orbitals to a set of particle (unoccupied) orbitals. In the present calculations, we considered all 13 valence occupied orbitals as hole orbitals. We selected the 67 lowest MVOs as particle orbitals and 85 MVOs (composed by the 18 doubly occupied orbitals plus the 67 lowest energy MVOs orbitals) as scattering orbitals. Our present calculations were performed in the $\mathrm{C}_{2 v}$ symmetry group, which has four irreducible representations. Hence, following the procedure described above, we obtained 20496 CSFs in the $A_{1}$ symmetry, 20361 CSFs in the $B_{1}$ symmetry, 16749 in the $\mathrm{B}_{2}$ symmetry, and 16637 in the $\mathrm{A}_{2}$ symmetry, providing a total of 74243 CSFs. The present target description gives a dipole polarizability of $6.98 \AA^{3}$, in reasonable agreement with the experimentally determined value of $7.23 \AA^{3}$. $^{24}$ This is encouraging, as the dipole polarizability has been shown to play a significant role in the scattering dynamics of positron collisions with atoms and molecules. ${ }^{25,26}$

Considering that the present SMC calculations are limited to elastic scattering, the open-channel space $P$ only involves the target ground state such that $P=\left|\Phi_{0}\right\rangle\left\langle\Phi_{0}\right|$. As a consequence, the positronium formation channel as well as electronic and vibrational excitation, which may contribute to the grand total cross section (TCS), is not accounted for in our calculations. Therefore, we do not expect good agreement between our SMC results and the TCS data above the positronium formation threshold, which was estimated at $\Delta_{p}=2.08 \mathrm{eV}\left(\Delta_{p}=I P-6.8 \mathrm{eV}\right.$, where $I P$ is the ionization threshold, for which we use the experimental value of $8.88 \mathrm{eV}^{27}$ ).

As already mentioned, furan has a permanent dipole moment. In our calculations, we obtained a value of $0.79 \mathrm{D}$, which is in reasonable agreement with the experimental result of $0.69 \mathrm{D}$. In order to take the long-range dipole interaction into account in our calculations, we used the standard Born-closure on the scattering amplitude, ${ }^{28,29}$ which is rewritten as

$$
\begin{aligned}
f\left(\vec{k}_{i}, \vec{k}_{f}\right)= & f^{\mathrm{FBA}}\left(\vec{k}_{i}, \vec{k}_{f}\right)+\sum_{\ell=0}^{\ell_{\mathrm{SMC}}} \sum_{m=-\ell}^{+\ell} \\
& \times\left[f_{\ell m}^{\mathrm{SMC}}\left(\vec{k}_{i}, k_{f}\right)-f_{\ell m}^{\mathrm{FBA}}\left(\vec{k}_{i}, k_{f}\right)\right] Y_{\ell m}^{*}\left(\hat{k}_{f}\right) .
\end{aligned}
$$

In this equation, $f^{\mathrm{FBA}}$ is the dipole potential scattering amplitude obtained within the first Born approximation (FBA) in the laboratory-fixed frame reference, $f_{\ell m}^{\mathrm{FBA}}$ is obtained by expanding the outgoing $\left(\vec{k}_{f}\right)$ angular dependence of $f^{\mathrm{FBA}}$ in spherical harmonics $\left(Y_{\ell m}\right)$, and $f_{\ell m}^{\mathrm{SMC}}$ is obtained from a similar expansion of the SMC scattering amplitude also in the laboratory-fixed frame. The lower partial waves that account for the short-range interactions are 
described within the SMC framework, while the FBA approximation to the dipole potential accounts for the higher partial waves $\left(\ell_{\max }<\ell<\infty\right)$. The upper limit for the SMC partial-wave expansion $\left(\ell_{\max }\right)$ depends on the collision energy and was chosen to provide the best possible agreement between DCSs obtained with and without the Born-closure procedure at high scattering angles. In the present furan calculations, we chose $\ell_{\max }=1$ from $0.1 \mathrm{eV}$ to $0.5 \mathrm{eV}, \ell_{\max }=2$ from $0.6 \mathrm{eV}$ to $2.0 \mathrm{eV}, \ell_{\max }=3$ from $2.5 \mathrm{eV}$ to $3.5 \mathrm{eV}, \ell_{\max }=4$ from $4.0 \mathrm{eV}$ to $4.5 \mathrm{eV}, \ell_{\max }=5$ from $5.0 \mathrm{eV}$ to $9.5 \mathrm{eV}$, and $\ell_{\max }=6$ from $10 \mathrm{eV}$ to $20 \mathrm{eV}$.

\section{B. Independent atom model with screening corrected additivity rule}

The screening corrected additivity rule within the framework of the independent atom model (IAM-SCAR), as used in this study, has been described in previous publications. ${ }^{30,31}$ It has been successfully applied in other studies for several biologically relevant molecules (see Ref. 9 and the references therein) typically in the range of $0.1 \mathrm{eV}-10000 \mathrm{eV}$ incident energy. IAM-SCAR essentially treats the molecule as the sum of its constituent atoms, with scattering calculated using the optical potential method, ${ }^{32}$ initially applied to the constituent atoms of the molecule, i.e., $\mathrm{C}, \mathrm{H}$, and $\mathrm{O}$ in the case of furan. The atomic scattering potential is then represented by

$$
V(r)=V_{s}(r)+V_{p}(r)-i V_{a}(r) .
$$

The real part of Eq. (8) drives the elastic scattering dynamics and includes the electrostatic $\left[V_{s}(r)\right]$ and polarization $\left[V_{p}(r)\right]$ interactions. The imaginary part $\left[V_{a}(r)\right]$ describes all inelastic processes that are considered as absorptions from the incident positron beam. Owing to the last term in Eq. (8), the optical model potential method yields a complex phase shift $\delta_{l}=\lambda_{l}+i \mu_{l}$. This allows for the calculation of the atomic scattering amplitudes, from which the corresponding differential and integral elastic as well as the integral inelastic and, therefore, the total cross sections are derived.

The static potential was obtained from the charge density derived from Hartree-Fock atomic wave functions, using a procedure analogous to that of Reid and Wadehra. ${ }^{33}$ The dipole plus quadrupole polarization potential was developed from that reported by McEachran et $a l .^{34}$ for Ne but scaled by constants in order to match the known dipole and quadrupole polarizabilities of the $\mathrm{C}$, $\mathrm{H}$, and $\mathrm{O}$ atoms (see Ref. 30 for details). The absorption potential accounts for the discrete electronic excitations, positronium formation, and direct ionization. However, owing to the challenging nature of representing the Ps formation channel, the definition of the threshold energy for the absorption potential can be critical. Our recent improvements to the treatment of Ps formation have been outlined in detail previously. ${ }^{9,31}$ In brief, we maintain the energy dependent threshold $\Delta(E)$ defined in Ref. 30 by necessarily coinciding with the well-known Ps formation threshold of $\Delta_{p}=I-6.8 \mathrm{eV}$ (where $I$ is the ionization threshold) for lower energies and the lowest optically allowed excitation transition $\Delta$ for higher impact energies, but applying the smooth transition in threshold energy from low to high impact energy, as proposed in Ref. 9,

$$
\Delta(E)=\Delta-\left(\Delta-\Delta_{p}\right) /\left[1+\left(\frac{E}{3 I}-1\right)^{2}\right] .
$$

One consequence of this approach is that the positronium formation threshold is calculated relative to the atomic thresholds in the constituent atoms, which is typically somewhat lower than the molecular threshold. This affects the accuracy in the low energy region for this cross section, but once the positronium formation cross section peaks, we find that this calculation tends to work well. ${ }^{9}$ Once we have calculated the atomic scattering amplitudes, the IAM-SCAR procedure $^{7}$ gives the molecular scattering amplitudes, $F(\theta)$, from those of the constituent atoms, $f_{i}(\theta)$, according to the following expression:

$$
F(\theta)=\sum_{\text {atoms }} f_{i}(\theta) e^{i \vec{q} \cdot \vec{r}_{i}}
$$

where the momentum transfer is $\vec{q}=\vec{k}_{f}-\vec{k}_{i}$ and the atomic positions are given by $\vec{r}_{i}$. In this calculation, we incorporate the recent improvement of considering interference effects. ${ }^{35}$ This updated version is known as IAM-SCAR+I and basically provides the molecular differential cross section $\left(d \sigma_{\text {molecule }}^{\text {elastic }} / d \Omega\right.$ ) as a combination of the multicenter atomic amplitudes given by

$$
\begin{gathered}
\frac{d \sigma_{\text {molecule }}^{\text {elastic }}}{d \Omega}=\sum_{i j} f_{i}(\theta) f_{j}^{*}(\theta) \frac{\sin q r_{i j}}{q r_{i j}} \\
=\sum_{i}\left|f_{i}(\theta)\right|^{2}+\sum_{i \neq j} f_{i}(\theta) f_{j}^{*}(\theta) \frac{\sin q r_{i j}}{q r_{i j}} .
\end{gathered}
$$

Here, the interference term is the second summation in Eq. (12). In this case, $q \equiv|\vec{q}|=2 k \sin (\theta / 2)$ is the momentum transfer and $r_{i j}$ is the distance between atoms $i$ and $j$. By integrating Eq. (12), the corresponding molecular integral cross sections are represented by

$$
\sigma_{\text {molecule }}^{\text {total }}=\sum_{\text {atoms }} s_{i} \sigma_{\text {atom } i}^{\text {total }}+\sigma^{\text {interference }} .
$$

The factor $s_{i}$ is a screening correction, reducing the contribution of each atom to the total molecular cross section $\left(0 \leq s_{i} \leq 1\right)$ based on the position of the atom within the molecule. The correction factors can be calculated for each incident electron energy as a function of the corresponding atomic cross section at that energy and the geometrical overlapping of this cross section with those of the surrounding atoms in the molecule. This accounts for the fact that as the energy of the incoming particle decreases, the atomic cross sections overlap requiring a reduction of their relative contribution to the summation. This has the effect of extending the range of validity of the IAM method well below $100 \mathrm{eV},{ }^{32}$ although it does not take into account the specifics of the molecular structure and excitations in the scattering process. The remaining term in Eq. (13) is determined from

$$
\sigma^{\text {interference }} \equiv \int d \Omega \sum_{i \neq j} f_{i}(\theta) f_{i}^{*}(\theta) \frac{\sin q r_{i j}}{q r_{i j}} .
$$

Including interference terms in the calculation of both integral and differential cross sections for molecular targets eliminates 
an inconsistency between the differential and integral cross section values, which is inherent to the IAM-SCAR method. ${ }^{36}$ This means that no additional normalization procedure is required by the IAM-SCAR+I approach in order to fulfill the optical theorem.

\section{Dipole rotational excitations}

From the above description of the IAM-SCAR+I procedure, it is obvious that vibrational and rotational excitations are not considered in this calculation. However, for polar molecules such as furan, additional dipole-induced excitation cross sections can be calculated following the procedure suggested by Jain. ${ }^{37}$ Basically, it calculates differential and integral rotational excitation cross sections for a free electric dipole in the framework of the first Born approximation (FBA), which can be incorporated to our IAM-SCAR+I calculation in an incoherent way, just adding the results as an independent channel. Although rotational excitation energies are, in general, very low (typically a few $\mathrm{meV}$ ) in comparison with the incident electron energies, in order to validate the Born approximation, the latter energies should be higher than about $10 \mathrm{eV}$. Under these circumstances, rotational excitation cross sections $J \rightarrow J^{\prime}$ were calculated by weighting the population for the $J$ th rotational quantum number at $300 \mathrm{~K}$ and estimating the average excitation energy from the corresponding rotational constants. This method has been successfully used for other polar molecules (see Ref. 38 and the references therein). Additionally, when the permanent dipole moment of the molecule is relatively large, the FBA also fails for medium and large scattering angles. In order to partially address this situation, we introduced a correction based on that suggested by Dickinson, ${ }^{39}$ which brings a substantial improvement for positron (and electron) scattering cross sections with strongly polar molecules. This procedure introduces a first-order corrective term to the differential cross sections $\left(\frac{d \sigma^{D c k}}{d \Omega}\right)$ for medium and large angles while maintaining the FBA correction $\left(\frac{d \sigma^{B}}{d \Omega}\right)$ for lower angles,

$$
\begin{gathered}
\frac{d \sigma^{B}}{d \Omega} \approx \frac{\mu^{2}}{6 E_{i}} \frac{1}{\sin ^{2}(\theta / 2)}, \quad \theta<\theta_{c}, \\
\frac{d \sigma^{D c k}}{d \Omega} \approx \frac{\pi \mu}{64 E_{i}} \frac{1}{\sin ^{3}(\theta / 2)}, \quad \theta>\theta_{c},
\end{gathered}
$$

where $\mu$ is the permanent dipole moment of the molecule and $E_{i}$ is the energy of the projectile. In the case of furan, $\mu=0.69 \mathrm{D}$ and both curves smoothly join together at $\theta_{c}$, the critical angle at which they cross each other.

\section{RESULTS AND DISCUSSION}

The grand total cross section is shown in Fig. 1, where it is compared to the calculation from the IAM-SCAR+I theory as well as the theoretical calculation integrated over the relevant experimental angular range (as shown in Table I and the associated discussion). In general, the comparison between the experiment and theory is good and is somewhat better than the agreement observed in the case

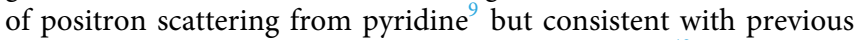
comparisons in positron scattering from pyrimidine. ${ }^{40}$ However, at energies below $5 \mathrm{eV}$, the experimental data are significantly higher in magnitude than the corrected theoretical predictions. This is to

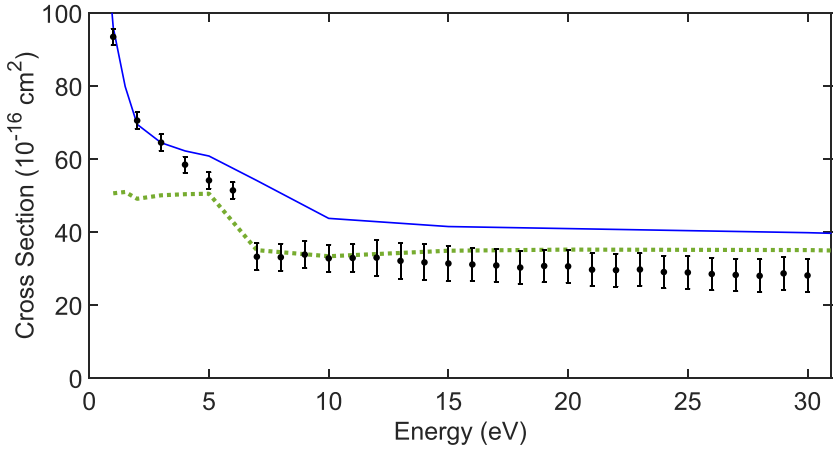

FIG. 1. Grand total cross section for positron scattering from furan. The black circles are the experimental data, and the blue solid line is the result using the IAM-SCAR+I approach. The green dotted line is the calculation, adjusted for the missing forward angles in the experiment. The apparent discontinuity at $7 \mathrm{eV}$ in the experimental data is due to the change in magnetic field to allow measurements of inelastic scattering, which affects the angular resolution. The jump in the adjusted calculation is due to the same change in resolution (see text and Table I for further details).

be expected, given that the calculation does not properly account for molecular structure effects in its approach, and these effects can be expected to play a stronger role at lower energies. In addition, it is clear that the IAM-SCAR + I calculation is missing the near-threshold part of the positronium formation cross section, as the threshold is calculated from the associated atomic states, as previously discussed (see Sec. III B). Given this, it may be said that the agreement at energies as low as $10 \mathrm{eV}$ is, in fact, quite remarkable given this theoretical approach and perhaps reflects the dominance of target dipole effects (including the dipole polarizability and the molecule's permanent dipole moment) in the scattering, which are reasonably modeled in the IAM-SCAR+I calculation.

A comparison of experimental and theoretical positronium formation cross sections is shown in Fig. 2 and again shows broad agreement. It can be seen that the theory has the threshold for this process at $5 \mathrm{eV}$, somewhat higher than the true threshold of $2.1 \mathrm{eV}$. We see that the maximum value of the cross section is similar in each case, at around $10 \times 10^{-16} \mathrm{~cm}^{2}$, but the peak positions are different, appearing lower in energy for the experimental measurement, at around $5 \mathrm{eV}$, compared to $10 \mathrm{eV}$ for the calculation. This is again likely due to the difference in threshold energy used for the calculation, which comes from using atomic thresholds in its phenomenological description of the positronium formation process (see Sec. III B). At higher energies, above $12 \mathrm{eV}$, the comparison between the experiment and theory is good, considering the experimental uncertainties, validating the theoretical approach used in this range.

The total inelastic cross section is shown in Fig. 3 and includes all electronic excitation and ionization processes, averaged over rotational and vibrational motion. In positron impact, due to the lack of the exchange interaction, we do not expect to be able to excite the low lying triplet electronic states of furan, rather the first accessible electronic state is at $6.04 \mathrm{eV}$ (the ${ }^{1} \mathrm{~B}_{2}$ valence state). We can see that there is some significant excitation of the electronic states of furan, before the ionization channel opens up at $8.89 \mathrm{eV}$. Comparison to the inelastic (electronic excitation plus ionization) 


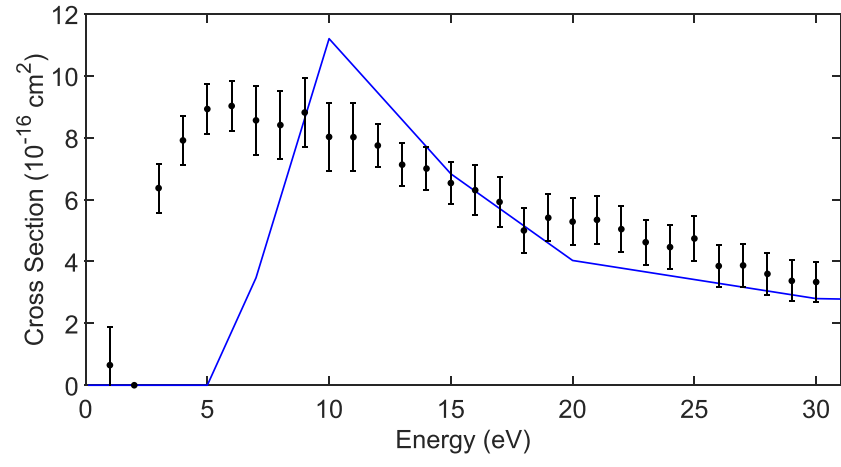

FIG. 2. Positronium formation cross section for positron scattering from furan. The black circles are the experimental data, and the blue line is the result using the IAM-SCAR+I approach. The apparent difference in the threshold energy is due to the phenomenological approach taken by the calculation to incorporate this process (see Sec. III B for further details).

part of the IAM-SCAR+I calculation is good, with the experiment lying a little lower in magnitude than the theory for the most part, although close to within the limits of the experimental error. Given the clear contribution from discrete electronic excitation below the ionization threshold, these excitations are also likely to still make a significant contribution to the measured total inelastic cross section above the ionization threshold.

Figure 4 shows the total elastic scattering cross section in the energy range from $1 \mathrm{eV}$ to $30 \mathrm{eV}$. Here, we can compare the experimental data to results from both the IAM-SCAR+I and SMC calculations of the scattering (note that the two features in the SMC calculation at energies between $5 \mathrm{eV}$ and $10 \mathrm{eV}$ are spurious structures). In the case of the IAM-SCAR+I theory, we have again presented an adjusted cross section, taking into account the missing angles from the experimental measurement. Above $6 \mathrm{eV}$, as previously noted, a reduced magnetic field was used in the detection region to separate the elastic and inelastic scattering contributions, which results in a different missing forward angle scattering contribution, as detailed in Table I. Due to the strong forward angle scattering, a result of the permanent dipole moment and dipole polarizability of this target,

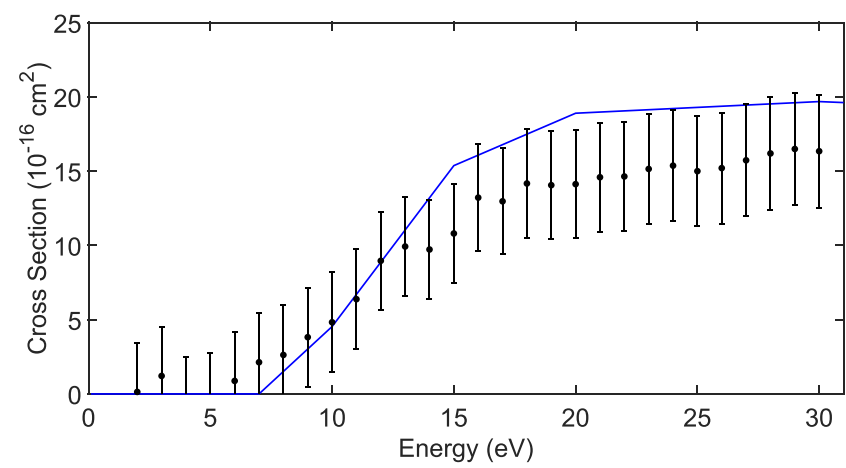

FIG. 3. Total inelastic scattering cross section for positrons incident on furan. The black circles are the experimental data, and the blue line shows the results using the IAM-SCAR+I method.

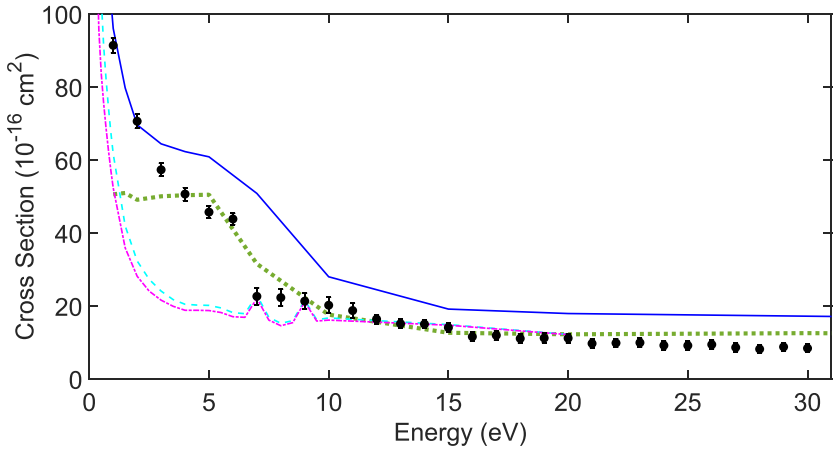

FIG. 4. Total elastic scattering cross section for positrons incident on furan. The black circles are the experimental data, the blue solid line shows the results using the IAM-SCAR+I method, and the green dotted line shows the IAM-SCAR+I theory adjusted for the missing experimental angles, as discussed in the text. The light blue dashed line and purple chain line are the results from the SMC theory with and without the Born closure included, respectively.

this appears as a "step" in the cross section between $6 \mathrm{eV}$ and $7 \mathrm{eV}$, corresponding to the increase in the angular range excluded from the experimental measurement. It can be seen that there is disagreement between the experiment and the SMC calculation below $10 \mathrm{eV}$, especially when considering that the experimental measurement misses the forward angle contribution to the total cross section and so can be expected to be lower in magnitude than the "true" cross section value. Given that agreement with the IAM-SCAR+I calculations is good at energies below this point, it seems clear that the positronium formation channel plays an important role here. Despite the fact that positronium formation is treated empirically in the IAMSCAR+I model, coupling between this and the elastic channel clearly plays a role in increasing the strength of the elastic channel. As positronium formation is not taken into account in the SMC calculation, the enhancement from this channel is missing. At higher energies, the experiment lies below the SMC data, which is more in line with expectations, and corresponds to the diminishing contribution of positronium formation to the total scattering cross section. As previously mentioned, comparison with the IAM-SCAR+I model is good below $20 \mathrm{eV}$, except at the very lowest energies. Again, in this case, the absence of positronium formation in the calculation in the energy range from $2 \mathrm{eV}$ to $5 \mathrm{eV}$ is likely to be the main reason for the discrepancy. At energies above $20 \mathrm{eV}$, the experimental data lie below the adjusted IAM-SCAR+I calculation, outside of the error limits of the measurement, suggesting that forward scattering is now being somewhat overestimated in the calculation. The data for the various total cross sections measured in this work are shown in Table II.

The data are presented in Figs. 5 and 6 for elastic differential cross sections in the energy range from $3 \mathrm{eV}$ to $15 \mathrm{eV}$. The numerical data for these measurements are also presented in Table III. The experimental cross sections are compared to the SMC and IAMSCAR $+\mathrm{I}$ calculation results as well as to corresponding experimental data for electron scattering from furan. ${ }^{41}$ The SMC theory is shown both with and without their Born closure technique applied. The theories and electron scattering measurements have been folded around $90^{\circ}$ to compare with the positron scattering measurements 
TABLE II. Data for the experimental total cross sections presented in this paper. The energy is given in $\mathrm{eV}$, and the cross sections and absolute uncertainties are in units of $10^{-16} \mathrm{~cm}^{2}$.

\begin{tabular}{|c|c|c|c|c|c|c|c|c|}
\hline \multirow{2}{*}{$\frac{\text { Energy }}{1}$} & \multicolumn{2}{|c|}{$\begin{array}{c}\text { Grand total } \\
\text { Cross section error }\end{array}$} & \multicolumn{2}{|c|}{$\begin{array}{c}\text { Total elastic } \\
\text { Cross section error }\end{array}$} & \multicolumn{2}{|c|}{$\begin{array}{c}\text { Ps formation } \\
\text { Cross section error }\end{array}$} & \multicolumn{2}{|c|}{$\begin{array}{c}\text { Total inelastic } \\
\text { Cross section error }\end{array}$} \\
\hline & 93.69 & 2.05 & 91.36 & 2.04 & 0.65 & 1.24 & & \\
\hline 2 & 70.75 & 2.01 & 70.56 & 1.98 & 0.00 & 0.80 & 0.14 & 3.32 \\
\hline 3 & 64.73 & 2.00 & 57.27 & 1.81 & 6.37 & 0.79 & 1.21 & 3.32 \\
\hline 4 & 58.67 & 2.00 & 50.62 & 1.78 & 7.91 & 0.80 & -0.80 & 3.31 \\
\hline 5 & 54.36 & 2.00 & 45.67 & 1.75 & 8.93 & 0.81 & -0.54 & 3.32 \\
\hline 6 & 51.63 & 2.00 & 43.79 & 1.55 & 9.03 & 0.81 & 0.88 & 3.32 \\
\hline 7 & 33.17 & 3.73 & 22.59 & 2.31 & 8.56 & 1.11 & 2.13 & 3.33 \\
\hline 8 & 33.03 & 3.73 & 22.24 & 2.29 & 8.41 & 1.10 & 2.62 & 3.33 \\
\hline 9 & 33.76 & 3.73 & 21.28 & 2.18 & 8.81 & 1.11 & 3.82 & 3.34 \\
\hline 10 & 32.73 & 3.73 & 20.16 & 2.14 & 8.03 & 1.10 & 4.83 & 3.35 \\
\hline 11 & 32.82 & 3.73 & 18.70 & 2.01 & 8.02 & 1.10 & 6.38 & 3.36 \\
\hline 12 & 32.93 & 4.90 & 16.36 & 1.23 & 7.75 & 0.70 & 8.96 & 3.30 \\
\hline 13 & 32.04 & 4.90 & 15.11 & 1.18 & 7.13 & 0.69 & 9.93 & 3.31 \\
\hline 14 & 31.63 & 4.89 & 14.98 & 1.18 & 7.00 & 0.68 & 9.72 & 3.31 \\
\hline 15 & 31.33 & 4.90 & 14.03 & 1.14 & 6.53 & 0.68 & 10.80 & 3.32 \\
\hline 16 & 31.05 & 4.48 & 11.53 & 1.28 & 6.30 & 0.82 & 13.22 & 3.58 \\
\hline 17 & 30.79 & 4.48 & 11.90 & 1.31 & 5.92 & 0.80 & 12.97 & 3.58 \\
\hline 18 & 30.21 & 4.48 & 11.04 & 1.26 & 5.00 & 0.74 & 14.17 & 3.64 \\
\hline 19 & 30.64 & 4.48 & 11.18 & 1.26 & 5.41 & 0.76 & 14.06 & 3.62 \\
\hline 20 & 30.54 & 4.48 & 11.13 & 1.26 & 5.29 & 0.76 & 14.13 & 3.63 \\
\hline 21 & 29.62 & 4.48 & 9.68 & 1.16 & 5.35 & 0.77 & 14.59 & 3.67 \\
\hline 22 & 29.50 & 4.48 & 9.81 & 1.18 & 5.04 & 0.75 & 14.64 & 3.68 \\
\hline 23 & 29.67 & 4.48 & 9.90 & 1.19 & 4.62 & 0.73 & 15.15 & 3.70 \\
\hline 24 & 28.99 & 4.48 & 9.15 & 1.14 & 4.46 & 0.72 & 15.37 & 3.73 \\
\hline 25 & 28.86 & 4.48 & 9.12 & 1.14 & 4.74 & 0.74 & 15.00 & 3.71 \\
\hline 26 & 28.44 & 4.48 & 9.39 & 1.17 & 3.85 & 0.68 & 15.21 & 3.74 \\
\hline 27 & 28.19 & 4.48 & 8.59 & 1.11 & 3.87 & 0.69 & 15.73 & 3.77 \\
\hline 28 & 27.94 & 4.48 & 8.15 & 1.07 & 3.60 & 0.68 & 16.19 & 3.80 \\
\hline 29 & 28.61 & 4.48 & 8.75 & 1.12 & 3.37 & 0.66 & 16.49 & 3.80 \\
\hline 30 & 28.05 & 4.48 & 8.37 & 1.09 & 3.34 & 0.66 & 16.34 & 3.81 \\
\hline
\end{tabular}

presented here (as outlined earlier). At the lowest energy of $3 \mathrm{eV}$, the experiment is considerably more forward peaked than either of the calculations or the electron scattering results. This is reflected in the grand total and total elastic cross sections presented in Figs. 1 and 4 , where the experimental data are significantly higher in absolute terms than the theoretical values. As previously discussed, it seems likely that this is due to the contribution of positronium formation to the scattering in this region, which is not properly incorporated in either calculation at this energy. It might be expected that dipole effects dominate the scattering at this energy, and so it is also instructive to compare to electron scattering results. Any dipole effects will be the same for positron and electron scattering so that differences may give insight into other important underlying scattering processes. Both theories have better agreement with the electron scattering data, again suggesting that positronium formation plays an important additional role in the positron scattering. The SMC calculation with Born closure aligns more closely the electron scattering experimental data at this energy, and it seems clear that the application of Born closure is required to properly account for the forward peaked nature of the scattering in this approach. This is true for all of the data presented in Figs. 5 and 6 and borne out by the forward peaking exhibited in the IAM-SCAR+I calculations. The two theories have much the same shape, although the IAM-SCAR+I calculation is higher in magnitude than the SMC theory for all angles and is in reasonable agreement with the experimental data angles above $20^{\circ}$. The large difference seen between the experimental positron and electron scattering data may also be partly related to the presence of the $\pi^{*}$ negative ion shape resonance at around $3 \mathrm{eV}$ in electron scattering, which has a strong effect on the collision dynamics around this energy, although the resonance is not present in the positron SMC calculation, which closely aligns with the experimental electron scattering measurements. At $5 \mathrm{eV}$, we find agreement between the experiment and the IAM-SCAR+I calculations is generally improved, and both datasets indicate more forward scattering than that is seen in the SMC theory, again probably related to the importance of positronium formation, which is starting to "turn on" at this energy in the IAM-SCAR+I theory. At angles above $20^{\circ}$, however, we see better agreement between the SMC calculation 

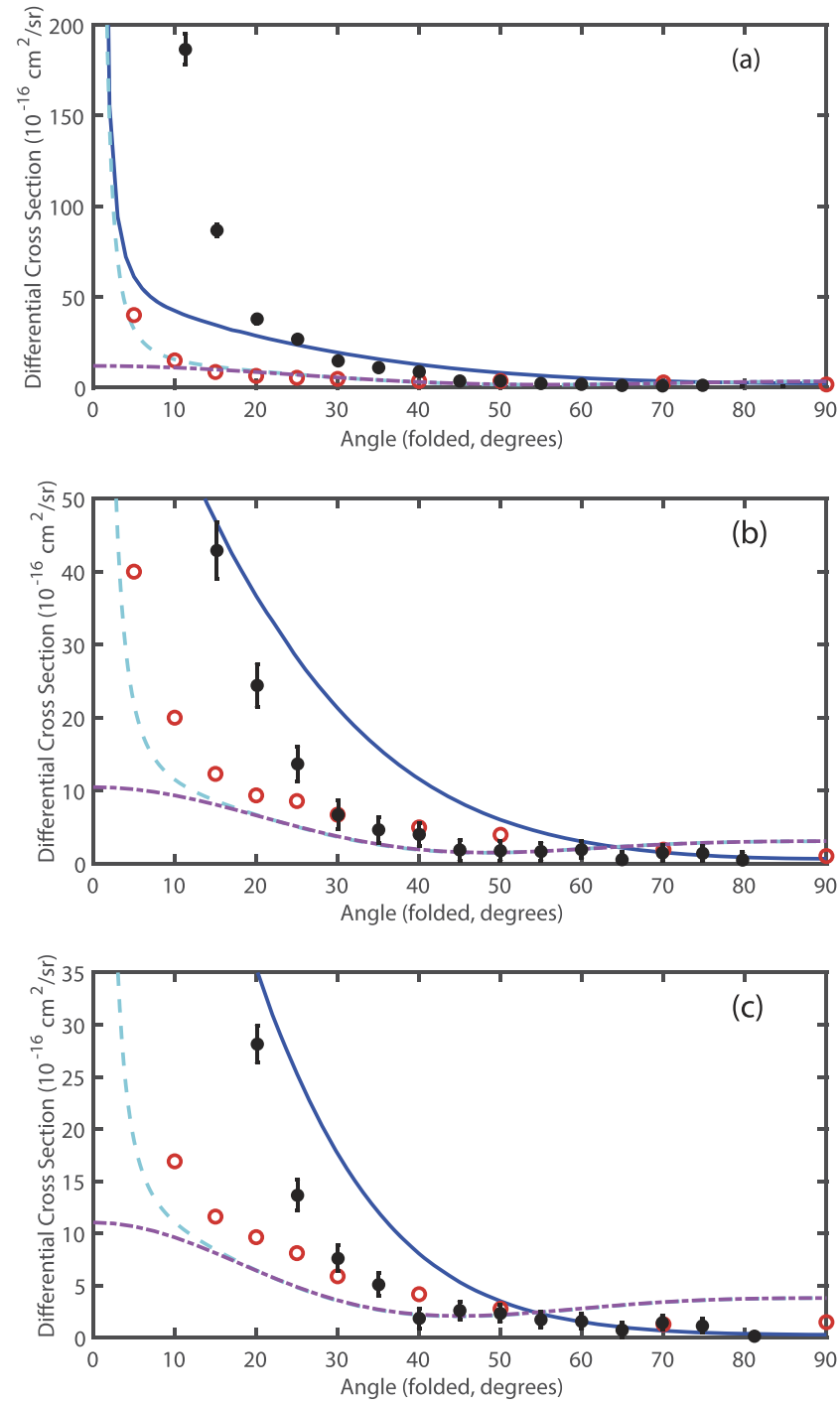

FIG. 5. Angular differential scattering of positrons from furan: (a) $3 \mathrm{eV}$, (b) $5 \mathrm{eV}$, and (c) $7 \mathrm{eV}$. The black points are the experimental data, and the open red circles are electron scattering data from Ref. 41. IAM-SCAR+I calculations are shown as blue lines, and the SMC calculation is shown as a blue dashed line and purple chain line, with and without the Born closure applied, respectively.

and the experimental data, with the experimental positron scattering more forward peaked than that observed for electron scattering. A similar analysis can be made at $7 \mathrm{eV}$ with the experimental data lying in between the two calculations, favoring the IAM-SCAR+I data at smaller scattering angles and the SMC approach from $30^{\circ}$ to $60^{\circ}$. In the highest part of the angular range, above $60^{\circ}$, agreement is best with the IAM-SCAR+I calculation. The experimental electron and positron scattering data are in much better agreement with each other at these energies, although there is still a discrepancy between the two for the most forward scattering angles. Agreement between the SMC positron scattering calculation and the electron scattering data again suggests that positronium must play
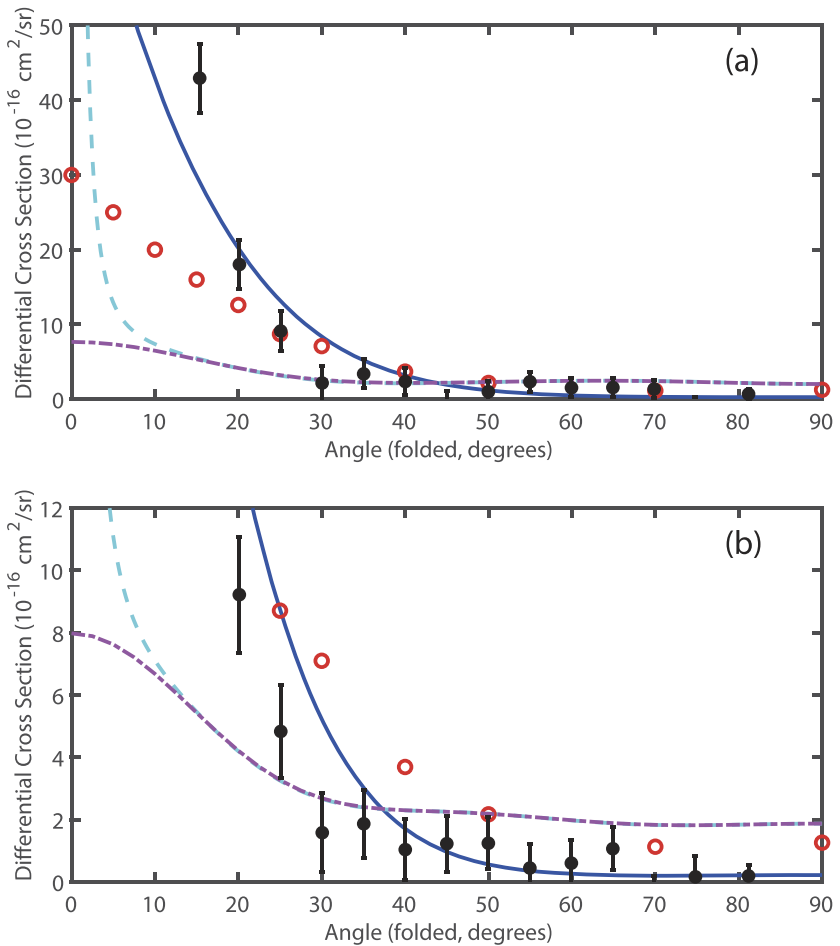

FIG. 6. Angular differential scattering of positrons from furan: (a) $10 \mathrm{eV}$ and (b) $15 \mathrm{eV}$. The black points are the experimental data, and the open red circles are electron scattering data from Ref. 41. IAM-SCAR+I calculations are shown as blue lines, and the SMC calculation is shown as a blue dashed line and purple chain line, with and without the Born closure applied, respectively.

a significant role in explaining the difference between this calculation and the present experimental data. It should be noted that the series of negative ion resonances in electron scattering, below $10 \mathrm{eV},{ }^{41}$ probably also plays some role in the differences seen here in the lowest range of energies. The data for $10 \mathrm{eV}$ and $15 \mathrm{eV}$ are presented in Fig. 6, and it should be noted that both of these energies lie in the region where the positronium formation, electronic excitation, and ionization channels are all open, with the contribution of positronium formation to the total scattering declining in relative importance. The experimental data tend to favor the results from the IAM-SCAR+I approach, with largely good agreement within the limits of the measurement across the entire angular range. The SMC calculation with Born closure applied still appears to underestimate the forward angle scattering, but agreement is good for angles above $25^{\circ}$ in both cases. With the ionization and positronium formation channels both open, it is to be expected that agreement between the SMC approach and experiment will be somewhat poorer. Agreement between the electron and positron scattering data improves further as the incident energy increases, with generally reasonable agreement at a scattering energy of $15 \mathrm{eV}$, when taking into account the experimental uncertainties. This observation suggests that the exchange interaction, which only exists for the case of electron scattering, is quite small in the elastic channel at these higher energies, and that the dipole scattering effects dominate. In addition, we can 
TABLE III. Data for the experimental differential cross sections presented in this paper. The angles are given in degrees, and the cross sections and absolute uncertainties are in units of $10^{-16} \mathrm{~cm}^{2} \mathrm{sr}^{-1}$.

\begin{tabular}{|c|c|c|c|c|c|c|c|c|c|c|}
\hline \multirow{2}{*}{$\frac{\text { Angle }}{85}$} & \multicolumn{2}{|c|}{$\begin{array}{c}3 \mathrm{eV} \\
\text { Cross section error }\end{array}$} & \multicolumn{2}{|c|}{$\begin{array}{c}5 \mathrm{eV} \\
\text { Cross section error }\end{array}$} & \multicolumn{2}{|c|}{$\begin{array}{c}7 \mathrm{eV} \\
\text { Cross section error }\end{array}$} & \multicolumn{2}{|c|}{$\begin{array}{c}10 \mathrm{eV} \\
\text { Cross section error }\end{array}$} & \multicolumn{2}{|c|}{$\begin{array}{c}15 \mathrm{eV} \\
\text { Cross section error }\end{array}$} \\
\hline & -0.30 & 0.81 & & & & & & & & \\
\hline 80 & -0.19 & 0.89 & 0.50 & 1.05 & 0.13 & 0.35 & 0.68 & 0.64 & 0.19 & 0.36 \\
\hline 75 & 1.26 & 0.91 & 1.42 & 1.05 & 1.12 & 0.65 & -0.85 & 1.18 & 0.16 & 0.67 \\
\hline 70 & 0.93 & 0.92 & 1.47 & 1.08 & 1.42 & 0.67 & 1.36 & 1.20 & -0.50 & 0.68 \\
\hline 65 & 1.19 & 0.95 & 0.55 & 1.11 & 0.72 & 0.69 & 1.56 & 1.24 & 1.07 & 0.70 \\
\hline 60 & 1.81 & 1.00 & 1.92 & 1.16 & 1.55 & 0.72 & 1.53 & 1.29 & 0.60 & 0.74 \\
\hline 55 & 2.26 & 1.05 & 1.67 & 1.23 & 1.71 & 0.76 & 2.33 & 1.36 & 0.45 & 0.78 \\
\hline 50 & 3.67 & 1.12 & 1.77 & 1.32 & 2.32 & 0.81 & 0.99 & 1.46 & 1.24 & 0.83 \\
\hline 45 & 3.62 & 1.22 & 1.88 & 1.43 & 2.59 & 0.87 & -0.55 & 1.60 & 1.23 & 0.90 \\
\hline 40 & 8.76 & 1.34 & 4.00 & 1.57 & 1.84 & 0.97 & 2.36 & 1.75 & 1.04 & 0.99 \\
\hline 35 & 10.99 & 1.50 & 4.64 & 1.75 & 5.08 & 1.08 & 3.39 & 1.94 & 1.87 & 1.10 \\
\hline 30 & 14.72 & 1.72 & 6.70 & 2.01 & 7.59 & 1.23 & 2.16 & 2.23 & 1.58 & 1.26 \\
\hline 25 & 26.67 & 2.02 & 13.66 & 2.38 & 13.64 & 1.46 & 9.12 & 2.67 & 4.83 & 1.50 \\
\hline 20 & 37.81 & 2.49 & 24.42 & 2.94 & 28.14 & 1.80 & 18.02 & 3.27 & 9.22 & 1.86 \\
\hline 15 & 86.71 & 3.27 & 42.90 & 3.86 & & & 42.95 & 4.60 & & \\
\hline 11 & 186.54 & 8.39 & & & & & & & & \\
\hline
\end{tabular}

infer that the influence of the positronium formation channel on the scattering dynamics is becoming diminished. While agreement between the positron and electron scattering data appears to be much better at higher scattering angles, for all energies, it is difficult to draw any definitive conclusions as to any interpretation due to the small magnitudes and hence proportionally large error bars on both measurements.

\section{CONCLUSION}

This paper has presented results from a joint experimental and theoretical study on the scattering of positrons from furan molecules at energies up to $30 \mathrm{eV}$. At the grand total cross section level, there is good agreement between the IAM-SCAR+I calculation and the experimental data, once the effect of missing forward angle scattering in the experimental measurements is allowed for. Differences arising in the positronium formation cross section are likely to arise from the phenomenological nature of its description in that theory, but agreement between the two remains quite reasonable. Some effects from this difference are apparent in the comparison between the experiment and theory. For the total inelastic cross section (electronic excitation plus ionization), agreement is good within the error bars on the experimental data. In all scattering channels, it is apparent that the chemical properties of the target, in particular the target dipole moment and dipole polarizability, play a key role in the scattering dynamics for furan. This is in line with previous work, which has demonstrated the importance of these properties in different aspects of positron scattering ${ }^{25,26,42}$ and even positron binding and annihilation.

In the case of elastic scattering, there are two theories presented, the IAM-SCAR+I and SMC calculations. Differences between the experiment and theory in the total elastic cross section can be attributed mainly to differences in the forward angle scattering. The experiment, in general, observes a larger amount of forward scattering than either of the theories. Agreement between all the data improves as the energies increase, although it is clear that differences in the calculations are affecting how the two theories predict the amount of forward angle scattering. It seems likely that the inclusion of positronium formation in the IAM-SCAR $+\mathrm{I}$ approach has an effect on this aspect of the scattering calculation, which is absent in the SMC formulation. A comparison is also made to electron scattering data, ${ }^{41}$ which is quite different to the positron scattering measurements at energies below $10 \mathrm{eV}$-somewhat surprisingly given the expected dominance of the dipole interactions with this target. However, the presence of negative ion resonances in electron scattering across most of the lower energy range of the differential cross section measurements presented here is likely to be one reason for this, along with the contribution of positronium formation to the positron scattering processes and exchange in the electron scattering processes.

It can be seen from the present study that there is fairly reasonable agreement between the measurement and theory for positron scattering from this target. Despite this, it is still clear that disagreement remains regarding some aspects of the scattering dynamics. Clearly, further work is required to improve both theoretical descriptions of the scattering process, with positronium formation remaining a difficult process to incorporate in an $a b$ initio fashion. Improvements in the accuracy of the experiments as well as better discrimination of the different open scattering channels may also help in being able to refine the understanding of both scattering from furan and also for application to more varied targets that will be of relevance to future modeling studies.

\section{ACKNOWLEDGMENTS}

The authors would like to thank Mr. Ross Tranter for his ongoing technical support of the experimental equipment used in the 
measurements presented here. The experimental side of this work was funded through the Australian Research Council Discovery Program (Grant No. DP190100696). F.B. and G.G. acknowledge partial financial support from the Spanish Ministerio de Ciencia, Innovación y Universidades (Project Nos. FIS2016-80440 and PID2019104727RB-C21) and CSIC (Project No. LINKA20085). G.M.M. and M.H.F.B. acknowledge support from the Brazilian Agencies Coordenação de Aperfeiçoamento de Pessoal de Nível Superior (CAPES) and Conselho Nacional de Desenvolvimento Científico e Tecnológico (CNPq). G.M.M. and M.H.F.B. also acknowledge computational support from CENAPAD-SP and Professor Carlos de Carvalho for computational support at LFTC-DFis-UFPR and LCPAD-UFPR.

\section{DATA AVAILABILITY}

The data that support the findings of this study are available from the corresponding author upon reasonable request.

\section{REFERENCES}

${ }^{1}$ W. J. Tattersall, D. G. Cocks, G. J. Boyle, M. J. Brunger, S. J. Buckman, G. García, Z. L. Petrović, J. P. Sullivan, and R. D. White, Plasma Sources Sci. Technol. 26, 045010 (2017).

${ }^{2}$ R. D. White, W. Tattersall, G. Boyle, R. E. Robson, S. Dujko, Z. L. Petrovic, A. Bankovic, M. J. Brunger, J. P. Sullivan, S. J. Buckman et al., Appl. Radiat. Isot. 83, 77 (2014).

${ }^{3}$ R. E. Robson, M. J. Brunger, S. J. Buckman, G. Garcia, Z. L. Petrović, and R. D. White, Sci. Rep. 5, 12674 (2015).

${ }^{4}$ C. Makochekanwa, A. Bankovic, W. Tattersall, A. Jones, P. Caradonna, D. S. Slaughter, K. Nixon, M. J. Brunger, Z. Petrovic, J. P. Sullivan et al., New J. Phys. 11, 103036 (2009).

${ }^{5}$ W. Tattersall, L. Chiari, J. R. Machacek, E. Anderson, R. D. White, M. J. Brunger, S. J. Buckman, G. García, F. Blanco, and J. P. Sullivan, J. Chem. Phys. 140, 044320 (2014).

${ }^{6}$ L. Chiari, E. Anderson, W. Tattersall, J. R. Machacek, P. Palihawadana, J. P. Sullivan, G. García, F. Blanco, R. P. McEachran, M. J. Brunger et al., J. Chem. Phys. 138, 074301 (2013).

${ }^{7}$ L. Chiari, P. Palihawadana, J. R. Machacek, C. Makochekanwa, G. García, F. Blanco, R. P. McEachran, M. J. Brunger, S. J. Buckman, and J. P. Sullivan, J. Chem. Phys. 138, 074302 (2013).

${ }^{8}$ E. K. Anderson, R. A. Boadle, J. R. Machacek, L. Chiari, C. Makochekanwa, S. J. Buckman, M. J. Brunger, G. García, F. Blanco, O. Ingolfsson et al., J. Chem. Phys. 141, 034306 (2014).

${ }^{9}$ D. Stevens, T. J. Babij, J. R. Machacek, S. J. Buckman, M. J. Brunger, R. D. White, G. García, F. Blanco, L. Ellis-Gibbings, and J. P. Sullivan, J. Chem. Phys. 148, 144308 (2018).

${ }^{10}$ J. P. Sullivan, A. Jones, P. Caradonna, C. Makochekanwa, and S. J. Buckman, Rev. Sci. Instrum. 79, 113105 (2008).

${ }^{11}$ J. P. Sullivan, S. J. Gilbert, J. P. Marler, R. G. Greaves, S. J. Buckman, and C. M. Surko, Phys. Rev. A 66, 042708 (2002).

${ }^{12}$ J. R. Machacek, E. K. Anderson, C. Makochekanwa, S. J. Buckman, and J. P. Sullivan, Phys. Rev. A 88, 042715 (2013).

${ }^{13}$ S. J. Gilbert, C. Kurz, R. G. Greaves, and C. M. Surko, Appl. Phys. Lett. 70, 1944 (1997).
${ }^{14}$ E. V. Gromov, A. B. Trofimov, N. M. Vitkovskaya, J. Schirmer, and H. Köppel, J. Chem. Phys. 119, 737 (2003).

${ }^{15}$ J. J. Oh, K. W. Hillig, R. L. Kuczkowski, and R. K. Bohn, J. Phys. Chem. 94, 4453 (1990).

${ }^{16}$ J. P. Sullivan, C. Makochekanwa, A. Jones, P. Caradonna, D. S. Slaughter, J. Machacek, R. P. McEachran, D. W. Mueller, and S. J. Buckman, J. Phys. B: At., Mol. Opt. Phys. 44, 035201 (2011)

${ }^{17}$ M. J. Brunger, S. J. Buckman, and K. Ratnavelu, J. Phys. Chem. Ref. Data 46, 023102 (2017).

${ }^{18}$ J. S. E. Germano and M. A. P. Lima, Phys. Rev. A 47, 3976 (1993).

${ }^{19}$ E. P. da Silva, J. S. E. Germano, and M. A. P. Lima, Phys. Rev. A 49, R1527 (1994).

${ }^{20}$ R. F. da Costa, M. T. D. N. Varella, M. H. F. Bettega, R. F. C. Neves, M. C. A. Lopes, F. Blanco, G. García, D. B. Jones, M. J. Brunger, and M. A. P. Lima, J. Chem. Phys. 144, 124310 (2016).

${ }^{21}$ R. F. da Costa, J. C. Ruivo, F. Kossoski, M. T. D. N. Varella, M. H. F. Bettega, D. B. Jones, M. J. Brunger, and M. A. P. Lima, J. Chem. Phys. 149, 174308 (2018).

${ }^{22}$ M. W. Schmidt, K. K. Baldridge, J. A. Boatz, S. T. Elbert, M. S. Gordon, J. H. Jensen, S. Koseki, N. Matsunaga, K. A. Nguyen, S. Su et al., J. Comput. Chem. 14, 1347 (1993).

${ }^{23}$ C. W. Bauschlicher, Jr., J. Chem. Phys. 72, 880 (1980).

${ }^{24}$ M. Gussoni, M. Rui, and G. Zerbi, J. Mol. Struct. 447, 163 (1998).

${ }^{25}$ A. Zecca, L. Chiari, A. Sarkar, and M. J. Brunger, New J. Phys. 13, 115001 (2011).

${ }^{26}$ A. Zecca, N. Moser, C. Perazzolli, A. Salemi, and M. J. Brunger, Phys. Rev. A 76, 022708 (2007).

${ }^{27}$ T. Veszprémi, L. Nyulászi, and J. Nagy, J. Organomet. Chem. 331, 175 (1987).

${ }^{28}$ M. T. N. Varella, M. H. F. Bettega, A. J. R. da Silva, and M. A. P. Lima, J. Chem. Phys. 110, 2452 (1999).

${ }^{29}$ I. I. Fabrikant, J. Phys. B: At., Mol. Opt. Phys. 49, 222005 (2016).

${ }^{30}$ L. Chiari, A. Zecca, S. Girardi, E. Trainotti, G. García, F. Blanco, R. P. McEachran, and M. J. Brunger, J. Phys. B: At., Mol. Opt. Phys. 45, 215206 (2012).

${ }^{31}$ F. Blanco, A. M. Roldán, K. Krupa, R. P. McEachran, R. D. White, S. Marjanović, Z. L. Petrović, M. J. Brunger, J. R. Machacek, S. J. Buckman et al., J. Phys. B: At., Mol. Opt. Phys. 49, 145001 (2016).

${ }^{32}$ F. Blanco and G. García, Phys. Rev. A 67, 022701 (2003).

${ }^{33}$ D. D. Reid and J. M. Wadehra, J. Phys. B: At., Mol. Opt. Phys. 29, L127 (1996).

${ }^{34}$ R. P. McEachran, A. G. Ryman, A. D. Stauffer, and D. L. Morgan, J. Phys. B: At. Mol. Phys. 10, 663 (1977).

${ }^{35}$ F. Blanco, L. Ellis-Gibbings, and G. García, Chem. Phys. Lett. 645, 71 (2016).

${ }^{36}$ F. Blanco and G. García, Phys. Lett. A 317, 458 (2003).

${ }^{37}$ A. Jain, J. Phys. B: At., Mol. Opt. Phys. 21, 905 (1988).

${ }^{38}$ A. Sieradzka, F. Blanco, M. C. Fuss, Z. Mašín, J. D. Gorfinkiel, and G. García, J. Phys. Chem. A 118, 6657 (2014).

${ }^{39}$ A. S. Dickinson, J. Phys. B: At. Mol. Phys. 10, 967 (1977).

${ }^{40}$ P. Palihawadana, R. Boadle, L. Chiari, E. K. Anderson, J. R. Machacek, M. J. Brunger, S. J. Buckman, and J. P. Sullivan, Phys. Rev. A 88, 012717 (2013).

${ }^{41}$ M. Khakoo, J. Muse, K. Ralphs, R. da Costa, M. Bettega, and M. Lima, Phys. Rev. A 81, 062716 (2010).

${ }^{42}$ J. R. Machacek, F. Blanco, G. Garcia, S. J. Buckman, and J. P. Sullivan, J. Phys. B: At., Mol. Opt. Phys. 49, 064003 (2016).

${ }^{43}$ J. R. Danielson, D. H. E. Dubin, R. G. Greaves, and C. M. Surko, Rev. Mod. Phys. 87, 247 (2015). 